Extended abstract for the technical paper to be presented at Supercomputing 2000

Conference to be held in Dallas, TX, November 4-10, 2000.

\title{
PARALLEL UNSTEADY TURBOPUMP SIMULATIONS FOR LIQUID ROCKET ENGINES
}

\author{
Cetin Kiris, Dochan Kwak, and William Chan \\ Numerical Aerospace Systems Simulation Division \\ NASA-Ames Research Center, Moffet Field, CA 94035
}

\section{ABSTRACT}

This paper reports the progress being made towards complete turbo-pump simulation capability for liquid rocket engines. Space Shuttle Main Engine (SSME) turbo-pump impeller is used as a test case for the performance evaluation of the MPI and hybrid MPI/Open-MP versions of the INS3D code. Then, a computational model of a turbo-pump has been developed for the shuttle upgrade program. Relative motion of the grid system for rotor-stator interaction was obtained by employing overset grid techniques. Time-accuracy of the scheme has tion was obtained by employing overset grid techniques. Thutations for SSME turbo-pump,
been evaluated by using simple test cases. Unsteady computation
which contains 136 zones with 35 Million grid points, are currently underway on Origin 2000 systems at NASA Ames Research Center. Results from time-accurate simulations with moving boundary capability, and the performance of the parallel versions of the code will be presented in the final paper.

\section{INTRODUCTION}

The motivation of this effort is based on two primary elements. First, the entire turbopump simulation intends to provide a computational framework for design and analysis for the liquid rocket engine fuel supply system. This effort is part of the High Performance Computing and Communications (HPCC) advanced technology application projects. Second objective of this research is to support the design of liquid rocket systems for the Advanced Space Transportation System. Since the space launch systems in the near future are likely to rely on liquid rocket engines, increasing the efficiency and reliability of the engine components is an important task. One of the major problems in the liquid rocket engine is to understand fluid dynamics of fuel and oxidizer flows from the fuel tank to plume. Understanding the flow through the entire turbopump geometry through numerical simulation will be of significant value toward design. This will help to improve the safety of future space missions.

The objective of this paper to present the capability and the accuracy of three-dimensional (3D) computational fluid dynamics (CFD) methods as efficient design analysis tools on high performance computer platforms. A substantial computational time reduction for these 3D unsteady flow simulations will be able to reduce design cycle time of the rocket engine turbo-pumps. One of the HPCC/CAS milestones is to reach 5 times speed up in year 2001 compare to the state-of-the-art technology available in 1999. Part of this speed up will be due to enhancements in computer hardware platforms. Remaining por- 
tion of the speed-up will be contributed by advances in algorithms and by efficient parallel implementations. The following section outlines the initial effort and steps taken in order to meet this milestone.

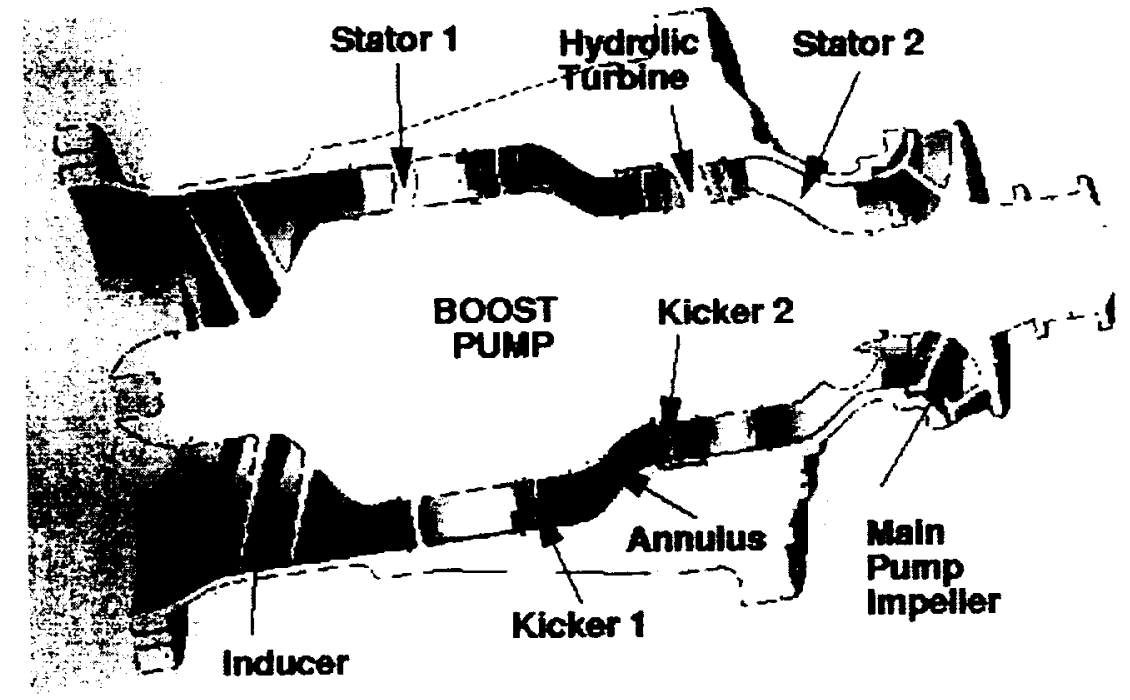

Figure 1. Schematic view of RSTS boost pump and its components.

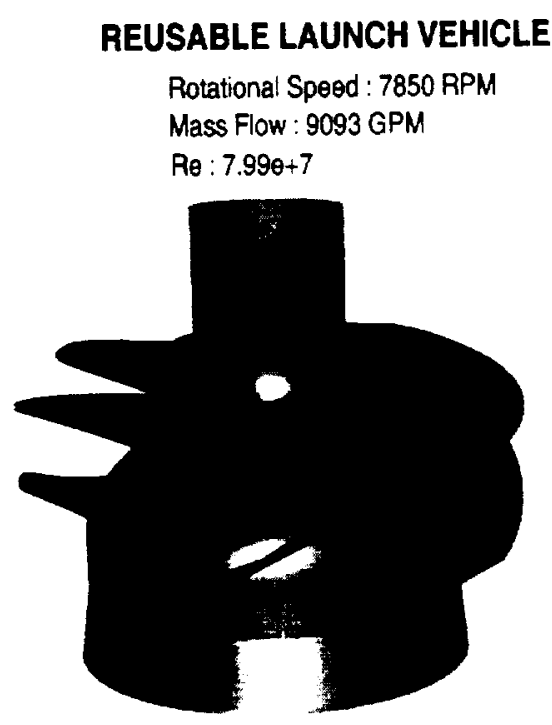

Geometry

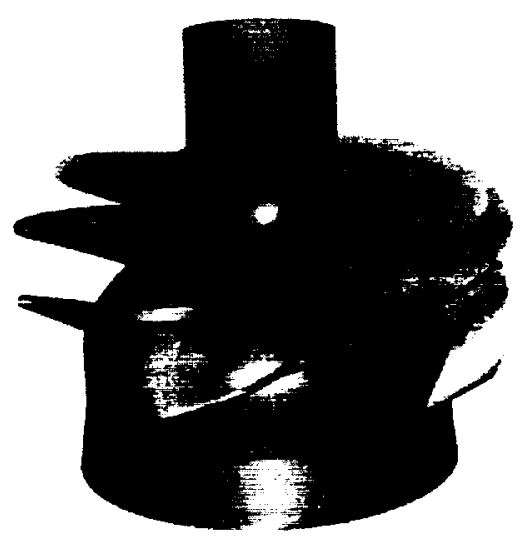

Surface Pressure

Figure 2. Geometry and surface pressure for the boost pump inducer

\section{APPROACH AND RESULTS}

The current geometry for the LOX boost turbopump has various rotating and stationary components, such as inducer, stators, kicker, hydrolic turbine, where the flow is exteremly unsteady. The components of the entire turbopump are plotted in Figure 1. Figure 2 shows the geometry and computed surface pressure of the inducer. The inducer and the 
hydraulic turbine rotate in different rotational speed. This causes severe unsteady interactions between rotating and stationary parts. To handle the geometric complexity and moving boundary problems, overset grid scheme is incorporated with the solver that new connectivity data is obtained at each time step. The Chimera overlapped grid scheme allows sub-domains move relative to each other, and provides a great flexibility when the boundary movement creates large displacements. In order to validate the current unsteady solution procedure, SSME shuttle upgrade pump configuration has been selected for validation purpose. Figure 3 shows the geometry of the test rig for this pump being tested at NASA-MSFC facilities. In this particular configuration, SSME impeller is unshrouded.

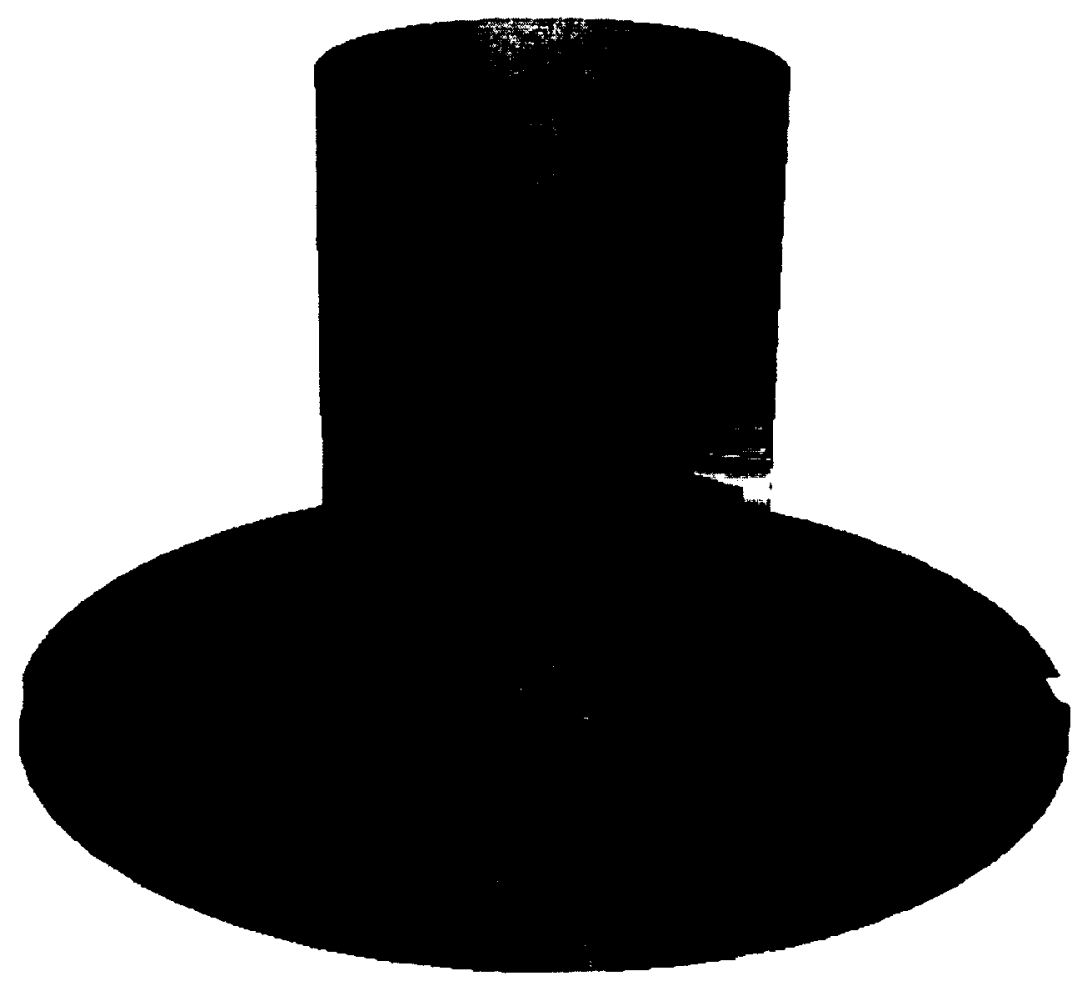

Figure 3.Geometry of SSME shuttle upgrade pump impeller

The development of the MPI, hybrid MPI/Open MP versions of the INS3D code is completed, and MLP version of the code is currently underway. The serial version of INS3D code is a multidimensional incompressible Navier-Stokes solver based on overset grid technology. The algorithm is based on the pseudocompressibility method as developed by Chorin ${ }^{1}$. The pseudocompressibility algorithm introduces a time-derivative of the pressure term into the continuity equation; the elliptic-parabolic type partial differential equations are transformed into the hyperbolic-parabolic type. The original version of the INS3D code $^{2}$ with pseudocompressibility approach utilized the Beam-Warming approximate factorization algorithm ${ }^{3}$ and central differencing of the convective terms. Since the convective terms of the resulting equations are hyperbolic, upwind differencing can be applied to these terms. The current versions of the INS3D code ${ }^{4}$ use flux-difference splitting based on the method of $R_{o} e^{5}$. The upwind differencing leads to a more diagonal dominant system than does central differencing and does not require a user specified artificial dissipation. The viscous flux derivatives are computed by using central differencing. In the steady-state formulation, the time derivatives are differenced using the Euler backward formula. The equations are solved iteratively in pseudo-time until the solution converges to a steady state. In the time-accurate formulation, the time derivatives in the momentum 
equations are differenced using a second-order, three-point, backward-difference formula. After the discretization in time, the pseudocompressibilty term and pseudo-time level are introduced to equations. The equations are iterated to convergence in pseudotime for each physical time step until the divergence of the velocity field has been reduced below a specified tolerance value. The matrix equation is solved iteratively by using a nonfactored Gauss-Seidel type line-relaxation scheme ${ }^{6}$, or GMRES scheme ${ }^{7}$ which maintains stability and allows a large pseudo-time step to be taken. Details of the numerical method can be found in Reference 4.
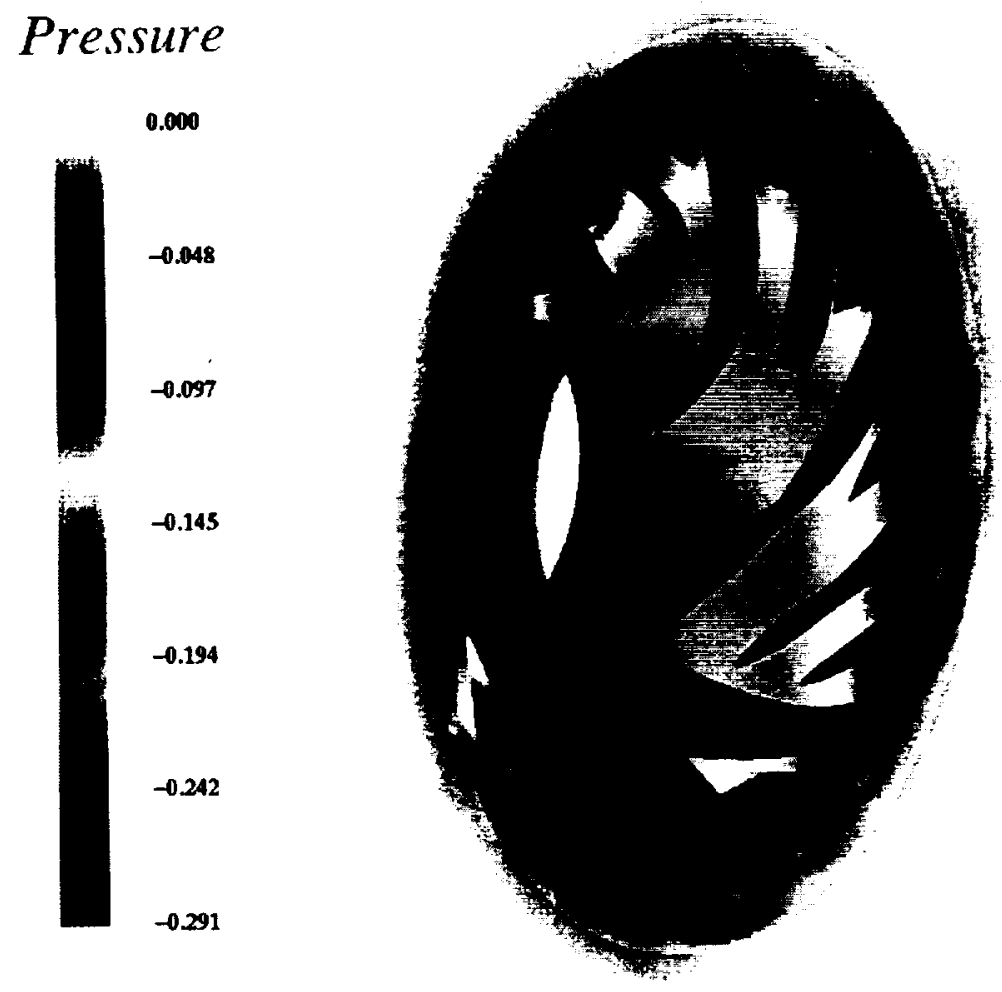

Figure 4. Computed surface pressure for SSME-HPFT impeller.

INS3D-MP| ${ }^{8}$ is based on the explicit massage-passing interface across processors and is primarily suited for distributed memory systems. The primary strategy is to distribute the zones across a set of processors. During the iteration, all the processors would exchange boundary condition data between processors whose zones shared interfaces with zones on other processors. A simple master-worker architecture was selected because it is relatively simple to implement and it is a common architecture for parallel CFD applications. All l/O was performed by master MPI process and data was distributed to the workers. After the initialization phase is complete, the program begins its main iteration loop. Computed surface pressure of the shrouded SSME impeller is shown in Figure 4. The solution is obtained by using INS3D-MPI with line-relaxation scheme. Computational grid for this case has 24 zones and 2.8 Million grid points. The MPI version of the code has coarse grain parallelism, and the number of processors is limited by the number of zones. Figure 5 shows Mflops for this computation on SGI Origin 2000 platform. The average speedup, 
as compared to the machine's ideal speedup, is about $65 \%$ for 24 processors. In MPIOpenMP hybrid version of the code, OpenMP directives are used for fine grain parallelism. Figure 6 shows time per iteration versus number of processors code. The cases for 4,12 , and $24 \mathrm{MPI}$ groups were used. Multiplication of the number of number of threads, such as $1,2,4,8$, and 16, w to number of processors. When number of threads and the number of MPl group is eq of the code slows down because the grid size threads is high is relatively small for higher number of threads.

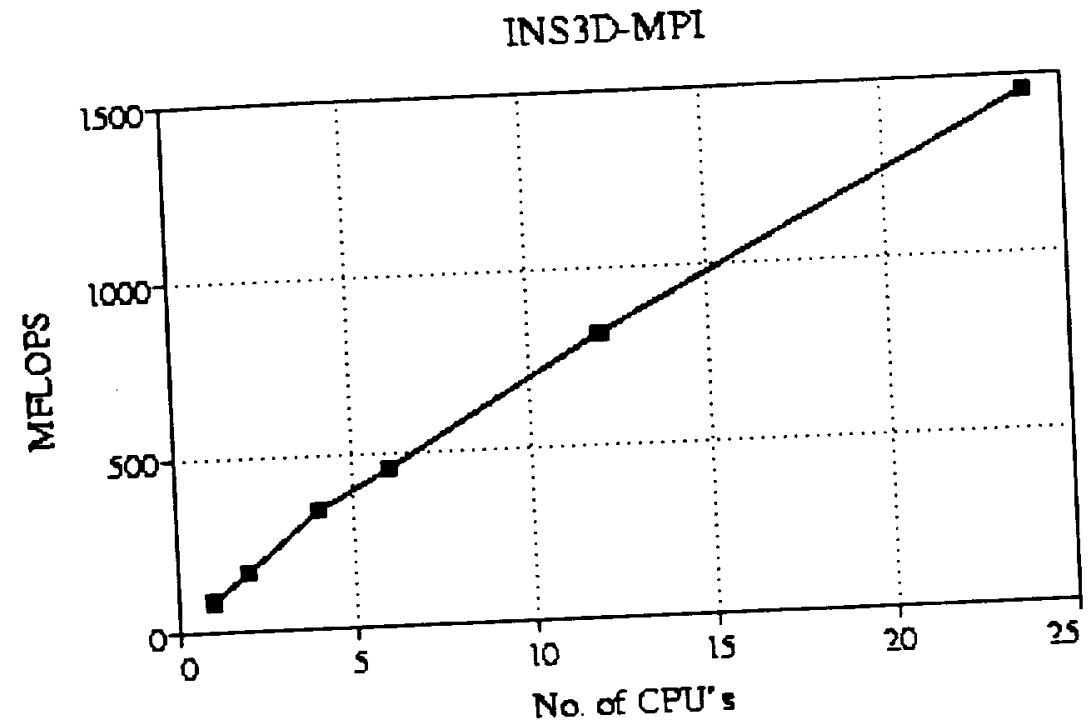

Figure 5 INS3D-MPI performance in the SGI O2K, SSME-HPFT Impeller.

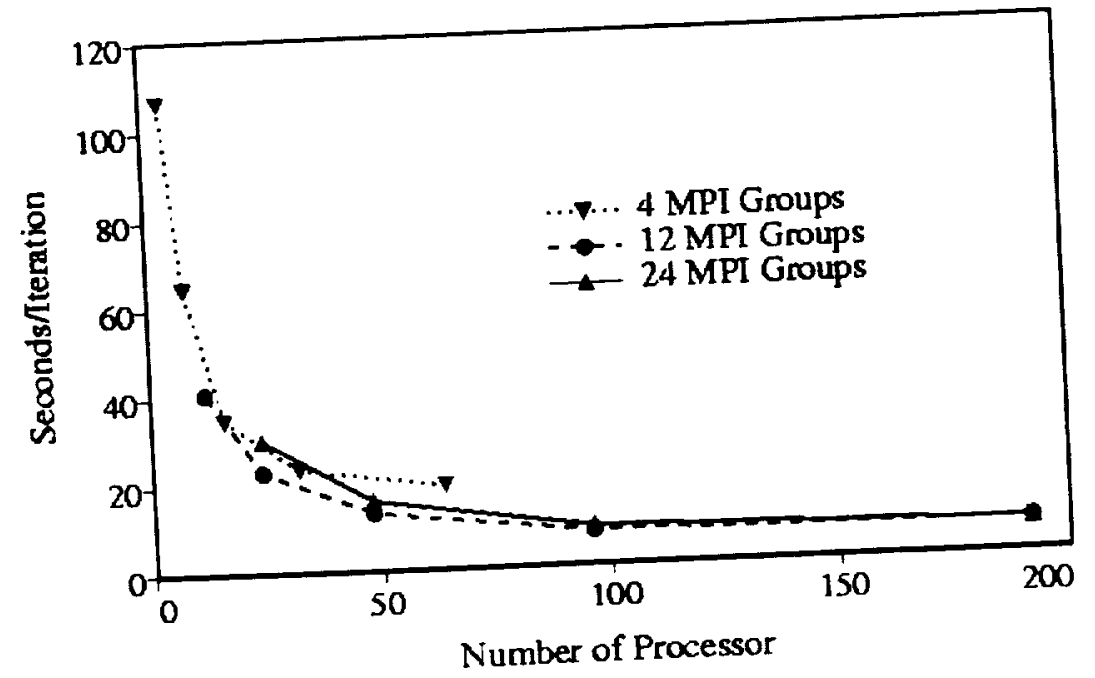

Figure 6 INS3D-MPI /Open-MP performance in the SGI O2K, SSME-HPFT Impeller.

The third parallel version of the code has been coded and in debugging stage. The shared memory Multi-Level Parallelism (MLP) technique developed at NASA Ames 政 zone parallelism with MLP, and intra zone parallelism is obtained by using OpenMP directives. Computational model for unsteady turbo-pump computations has been completed by using an overset grid technology. Figure 7 shows the overset grid surfaces. 
This particular impeller configuration has 60 zones with 19.5 Million grid points. Including inlet guide vane and diffuser blades, the computational model contains 136 zones with 35 Million grid points. Impeller blades rotate relative to inlet guide vanes and diffuser blades.

This configuration will be computed with INS3D-MLP code on 512 processors SGI Origin 2000 platform at NASA Ames are data storage, feature extraction, and overset lation, some of the additional challenges are data storage, developed at NASA-Ames is
connectivity synchronization. Data compression algorithm dincorporated in the procedure. Overset connectivity code (OVERFLOW-D ${ }^{11}$ ) will be run
incolo simultaneously with the flow solver.

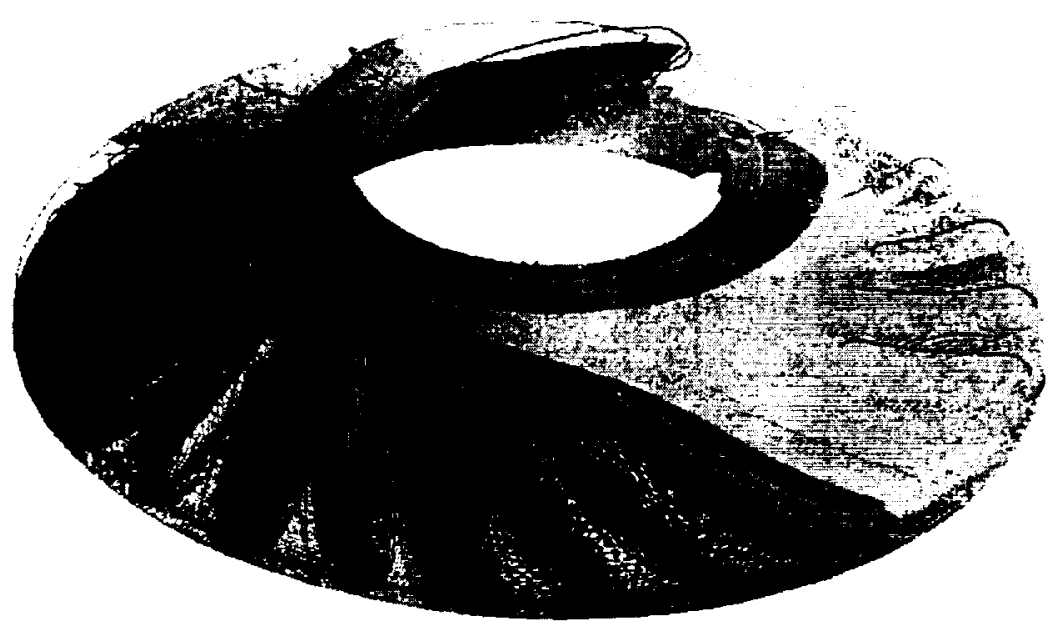

Figure 7. Computation grid of SSME impeller with 60 zones and 19.5 Million grid points

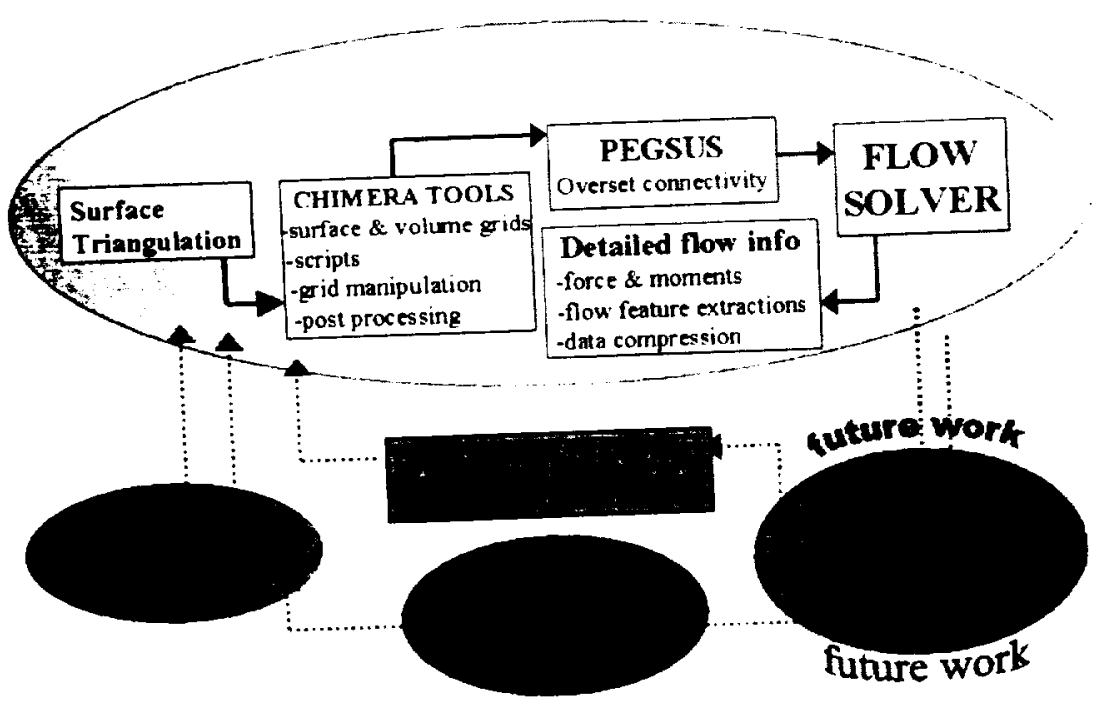

Figure 8. Flowchart from $C A D$ to flow feature extraction for the current procedure

Figure 8 shows a typical flowchart for the computational procedure from the CAD model to post-processing. In order to achieve unsteady simulation capability for the entire turbopump configuration, several key areas where the advancements are currently underway are outlined. 
Results from benchmark problems are presented for INS3D-MPI and MPI/OpenMP calculations. INS3D-MLP calculations with 35 Million grid points will be presented in the final paper.

\section{REFERENCES}

1. Chorin, A., J., "A Numerical Method for Solving Incompressible Viscous Flow Problems" Computational Physics, Vol. 2, pp. 12-26, 1967.

2. Kwak, D., Chang, J. L C., Shanks, S. P., and Chakravarthy, S., “A Three-Dimensional Incompressible Navier-Stokes Flow Solver Using Primitive Varlables," AIAA Journal, Vol. 24, No. 3, pp. 390-396, 1977.

3. Beam, R. M., Warming, R. F., " An Implicit Factored Scheme for the Compressible Navier-Stokes Equations," AIAA Journal, Vol 16, pp. 393-401, 1978.

4. Rogers, S. E., Kwak, D. and Kiris, C., "Numerical Solution of the Incompressible Navier-Stokes Equations for Steady and Time-Dependent Problems," AIAA Journal, Vol. 29, No. 4, pp. 603-610, 1991.

5. Roe, P.L., “Approximate Riemann Solvers, Parameter Vectors, and Difference Schemes," J. of Comp. Phys., Vol. 43, pp. 357-372 1981.

6. MacCormack, R., W., "Current Status of Numerical Solutions of the Navier-Stokes Equations," AIAA Paper No. 85-0032, 1985.

7. Rogers, S. E., "A Comparison of Implicit Schemes for the Incompressible NavierStokes Equations and Artificial Compressibility," AIAA Journal , Vol. 33, No. 10, Oct.

1995.
8. Faulkner, T., and Mariani, J., "MPI Parallelization of the Incompressible NavierStokes Solver (INS3D). ht tp: / / www. nas . nasa.gov/ - Eaction Geraphical Interface,

9. Chan, W. M., OVERGRID -- A Unified Oover 2000

10. Suhs, N. E., and Tramel, R. W., PEGSUS 4.0 User;s Manual, Arnold Air Force Base, Tennesse.

11. Meakin, R., and Wissink, M., " Unsteady Aerodynamic Simulation of Static and Moving Bodies Using Scalable Computers," $14^{\text {th }}$ AIAA CFD Conference, Norfolk, VA, 1999. 